\title{
BMJ Open Effectiveness of an online SUpport PRogramme (SUPR) for older hearing aid users: study protocol for a cluster randomised controlled trial
}

\author{
Janine FJ Meijerink, ${ }^{1}$ Marieke Pronk, ${ }^{1}$ Bernadette Paulissen, ${ }^{2}$ Birgit I Witte, ${ }^{3}$ \\ Bregje van der Wouden, ${ }^{1}$ Vera Jansen, ${ }^{4}$ Sophia E Kramer ${ }^{1}$
}

To cite: Meijerink JFJ, Pronk M, Paulissen B, et al. Effectiveness of an online SUpport PRogramme (SUPR) for older hearing aid users: study protocol for a cluster randomised controlled trial. BMJ Open 2017;7:e015012. doi:10.1136/ bmjopen-2016-015012

- Prepublication history and additional material are available. To view these files, please visit the journal online (http://dx.doi.org/ 10.1136/ bmjopen-2016-015012)

Received 2 November 2016 Revised 9 March 2017 Accepted 22 March 2017

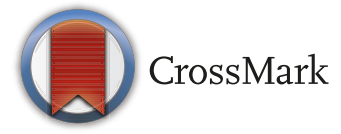

${ }^{1}$ Department of Otolaryngology, Head and Neck Surgery, Section Ear \& Hearing, Amsterdam Public Health Research Institute, VU University Medical Center, Amsterdam, The Netherlands ${ }^{2}$ AudioNova International B.V., Rotterdam, The Netherlands ${ }^{3}$ Department of Epidemiology and Biostatistics, VU University Medical Center, Amsterdam, The Netherlands

${ }^{4}$ Schoonenberg B.V., Dordrecht, The Netherlands

Correspondence to Janine FJ Meijerink; f. meijerink@vumc.nl

\section{ABSTRACT}

Background An educational SUpport PRogramme called SUPR has been developed for hearing aid users (HAUs) and their communication partners (CPs) offering care beyond hearing aid fitting. SUPR teaches its users communication strategies, hearing aid handling skills and personal adjustment to hearing impairment.

Methods/design Using a cluster randomised controlled trial design, 70 Dutch hearing aid dispenser practices were randomised into hearing aid fitting (care as usual, 34 practices) and hearing aid fitting including SUPR (36 practices). The aim was to recruit a total of 569 older (aged $50+$ years) first-time $(n=258)$ and experienced $(n=311)$ HAUs and their CPs. SUPR consists of a Practical Support Booklet and online material offered via email over a period of $6-7$ months. The booklet provides practical information on hearing aids, advice on communication strategies and home exercises. The online material consists of educational videos on hearing aid functionality and usage, communication strategies and peer testimonials. Finally, noncommittal email contact with the dispenser is offered. Every HAU is asked to assign a CP who is advised to be involved intensively. Effect measurements for HAUs and their CPs will occur at baseline and at 6, 12 and 18 months follow-up via online questionnaires. The primary outcomes for HAUs will be the use of communication strategies as measured by the subscales of the Communication Profile for the Hearing Impaired. A process evaluation will be performed.

Ethics and dissemination The study was approved by the Dutch Institutional Review Board of the VU Medical University Center Amsterdam. This intervention could contribute to lowering the hearing impairment burden in our ageing society. The results will be disseminated through peer-reviewed publications and scientific conferences.

Trial registration number ISRCTN77340339; Pre-results.

\section{BACKGROUND}

Hearing impairment is one of the most prevalent chronic health conditions affecting older adults. It was ranked fifth in the top 25 of global causes for years lived with disability in 2013. ${ }^{1}$ Due to the overall ageing of the population, ${ }^{2}$ the prevalence of hearing impairment

\section{Strengths and limitations of this study}

- This is the first study to evaluate the effects of an online educational SUpport PRogramme (SUPR) for hearing aid users that is implemented in a hearing aid dispensing (HAD) practice setting on a large scale.

- Hearing-impaired participants and their communication partners (CPs) originating from 70 HAD practices located all across the Netherlands were included.

- The online nature of the programme suits the current and future developments in the increasing internet use among the young-old (aged 55-74 years) and can reach out to those with reduced (physical) access to healthcare.

- The online nature might however reach a selective sample, especially among the oldest old (aged $75+$ years), who are willing or able to adopt the intervention (ie, only those with access to and willing to use the internet for this purpose).

- The study design does not allow the blinding of participants and researchers for intervention allocation. This could potentially lead to performance bias.

- The findings of the study will potentially contribute to improvement of hearing healthcare services for hearing-impaired people and their CPS.

is increasing rapidly, imposing a great burden on individuals and society.

Hearing impairment essentially leads to the inability to communicate effectively, which in turn can result in a cascade of effects leading to poor psychosocial outcomes such as loneliness, ${ }^{3-5}$ distress, ${ }^{6}$ depression ${ }^{67}$ and work-related fatigue. ${ }^{8}$ It has also been associated with accelerated cognitive decline ${ }^{9}$ and falls. ${ }^{10}$ The limitations on daily life activities and restrictions in social and societal participation that people experience depend on aspects that are both internal (such as age and applied coping styles) and external (such 
as availability of hearing aids, care facilities and social support) to the person. ${ }^{11}$ In addition, the level of impairment in hearing functions and structures is an important factor which can influence psychosocial outcomes. ${ }^{11}$

Partners and spouses can also be negatively affected by the hearing impairment of their loved ones. They generally experience frustration and embarrassment, for example, in challenging social communication settings. ${ }^{12}$ Communication difficulties in background noise, the partner's frequent request to repeat and the need to act as an interpreter may cause irritation and tension within a relationship. ${ }^{12}$ In a systematic review conducted by Kamil et al, it was found that communication partners (CPs, ie, spouses, partners, close family members, neighbours or caregivers) of people with hearing impairment experience decreased social functioning, poorer quality of life, and more participation restrictions than CPs of normally hearing individuals. ${ }^{13}$

The usual care provided for people with hearing impairment is often restricted to the assessment of hearing loss and the fitting of hearing aids. ${ }^{14}$ Hearing aid use has positive effects on quality of life, social and emotional well-being and may reduce depressive complaints ${ }^{15-17}$ and possibly even cognitive decline. ${ }^{18}$ Despite this abundant evidence on positive health effects, the uptake and use of hearing aids is low. It is estimated that around one-third of the adults who would benefit from hearing aids own them ${ }^{19-21}$ and $3 \%-20 \%$ of these owners never use them. ${ }^{22}{ }^{23}$ Reasons for low uptake and use have been investigated and include low perceived need of amplification reflected in low self-reported hearing disability, ${ }^{24-26}$ limited acceptance of hearing loss, ${ }^{24}$ low expectations of hearing aid benefits, ${ }^{2425}$ limited gain in noisy situations ${ }^{2526}$ and low overall sound quality. ${ }^{26}$ Other perceived barriers include stigma, ${ }^{25}{ }^{26}$ high monetary $\operatorname{costs}^{26}$ and the need for regular hearing aid care and maintenance. ${ }^{26}$ Finally, lack of social support or social pressure to get a hearing aid are factors having a negative impact on hearing aid use. $^{25} 26$

Given this broad spectrum of factors affecting hearing aid uptake and use, it has often been argued that hearing healthcare should not be restricted to the provision of hearing aids alone, but cover more than that to improve hearing aid success, everyday communication and wellbeing of hearing-impaired adults. ${ }^{27}$ This argument is in line with the biopsychosocial approach of health which is receiving increasing attention in the field of audiology: experienced hearing disability (ie, activity limitations and participation restrictions) is the outcome of a complex interaction between an individual and his/her contextual factors. ${ }^{28-30}$

Various interventions have been proposed in the past to complement hearing aid fitting. Examples are communication programmes aimed at improving speech perception and/or communication management. ${ }^{31}$ These programmes include speech perception training, communication management training and social support. $^{27} 3233$ For reviews, see Barker et al, Henshaw and Ferguson and Wong and Hickson. ${ }^{34-36}$ Examples of effective programmes are the Home Education programme $^{37}$ and the Active Communication Education group programme. ${ }^{38}$ Both programmes consist of modules on everyday communication situations, aiming to improve the use of communication strategies, personal adjustment to living with hearing impairment, quality of life, development of problem-solving skills and to decrease the level of experienced hearing disability. These programmes showed an improvement in communication strategies $^{37}$ and communicative participation restrictions and activity limitations. ${ }^{38}$

Communication training programmes, whether combined with hearing aid fitting or not, are rarely offered in hearing healthcare. ${ }^{27} 32$ When offered, there are various reasons why adults with hearing impairment would choose not to pursue communication training programmes; they could live in a rural area, have a lack of time or no easy access. ${ }^{32}$ The paradigm shift in healthcare from the traditional doctor-centric model to a more patient-centred model, combined with increasingly pervasive use of e-health methods and technology, means that the typical barriers causing the low use of (group) communication training programmes can now be overcome. ${ }^{39-41}$

A number of studies have recently been published reporting on the development and evaluation of online communication programmes. Thorén et al developed such a programme, ${ }^{42}$ which included reading material on hearing anatomy, hearing aids, communication strategies, assistive listening devices and guidelines for CPs. In addition, the intervention included weekly email contact with an audiologist, problem-solving exercises and online peer discussion on personal experiences with hearing loss. Thorén et al studied the effectiveness of the programme using a randomised controlled trial design in which the intervention group $(n=38)$ received the online programme, while the control participants $(n=38)$ were offered access to an internet discussion forum or were placed on a waiting list. ${ }^{42}$ The researchers found reduced symptoms of depression ${ }^{43}$ and a significant decrease of activity limitations and participation restrictions in the intervention group compared with the controls at 5 weeks directly after the intervention and at 3 months follow-up. ${ }^{42}$ Ferguson $e t$ al investigated the use of short interactive videos (reusable learning objects (RLOs)). ${ }^{44}$ RLOs were delivered via DVD for TV, computer and the internet and covered practical and psychosocial issues which are relevant for audiologic rehabilitation. The intervention group $(\mathrm{n}=103)$ received seven RLOs plus usual clinical services including hearing aid fitting and counselling. They were compared with a control group $(n=100)$ who received clinical services only and were placed on a waiting list. Participants in the intervention group had significantly better hearing aid skills and better knowledge on psychosocial issues than the control group after 7 weeks follow-up.

Where the online education programme of Thorén et $a l$ was evaluated in a sample of adults who were recruited 
by local advertisements and articles and were wearing a hearing aid for at least 1 year, ${ }^{42}$ Ferguson et al evaluated their RLOs in a small sample of patients of the audiology service of the Nottingham University Hospitals NHS Trust. Patients were adults who had been referred to the clinic by their family doctor. ${ }^{44}$ The participants in the study of Kramer et al mentioned earlier, were all patients of a specialised tertiary Audiology Centre, limiting the generalisability of the results. ${ }^{37}$ In the Netherlands, only a small number of hearing aid applicants receive hearing care through a tertiary clinic, that is, only those with relatively complex hearing problems. The vast majority of hearing aids are fitted in a dispenser practice.

To the best of our knowledge, there is no study available evaluating the effectiveness of an online communication training programme that is implemented on a large scale in a hearing aid dispensing (HAD) practice setting. This paper reports on the design of such a study. It addresses the different steps that will be taken to evaluate an online SUpport PRogramme (SUPR) for hearing-impaired adults and their CPs. SUPR is based on the Home Education programme developed by Kramer et al. ${ }^{37}$ The original version developed in 1995 has been updated so that it would be applicable for use over the internet. SUPR has also been expanded with extra elements including instructional videos on how to operate and maintain hearing aids and peer testimonials. All elements will be sent about biweekly via email.

This study aimed to involve seventy HAD practices, of which half will offer the training programme. This large number of practices contributes to a large sample size (and therefore statistical power), and it reflects real-world clinical practice and thus contributes to the external validity of the future results. The study will include an 18-month follow-up. As was mentioned earlier by Barker et al, Wong and Hickson and Kramer et al, more research on treatment efficacy in the long(er)-term is essential because it is possible that some short-term effects may disappear and other effects can arise. ${ }^{34637}$

The aim of this study is to determine the effectiveness of SUPR as part of standard HAD care among older hearing aid users (HAUs) and their CPs. Based on the active elements included in SUPR, we hypothesise that older HAUs who receive SUPR in addition to hearing aid fitting will show the following favourable effects at 18 months follow-up when compared with HAUs who receive hearing aid fitting only:

- More use of favourable and less use of unfavourable communication strategies (primary outcome measures).

- Better personal adjustment to hearing impairment, higher self-efficacy of hearing aid handling, higher hearing aid use, less activity limitations and participation restrictions, less handicap and disability, better self-reported intervention outcomes, higher readiness to do something about their hearing and higher satisfaction with HAD services (secondary outcome measures).
These effects will be studied both in first-time and experienced HAUs.

- Consistent with the findings by Kramer et $a l^{37}$ we hypothesise that effects on all outcomes will be larger in first-time HAUs than in experienced HAUs.

With regard to the CPs, we hypothesise that CPs who receive SUPR - as compared with CPs whose loved ones only receive hearing aid fitting-will show the following favourable effects:

- Lower third-party disability and better self-reported intervention outcomes.

\section{METHODS}

\section{Study design}

A cluster randomised controlled trial with an 18-month follow-up period will be performed. Cluster randomisation (with the HAD practice as a unit) was chosen over individual randomisation because the latter would hold a high risk of contamination. In case of individual randomisation, the HAD personnel would have to switch between approaches (SUPR/care as usual (CaU)) frequently and could accidentally refer to or offer SUPR to clients assigned to the $\mathrm{CaU}$ group. In addition, as the time between informing the clients about the study, receiving clients' consent and the start of SUPR/CaU was relatively short, performing randomisation on an individual level was not feasible. Dutch HAD practices and consequently all clients in these practices were randomly assigned to one of two groups. The control group received $\mathrm{CaU}$ which is hearing aid fitting only, while the intervention group received hearing aid fitting supplemented with SUPR.

\section{Care as usual}

CaU starts with a preparation appointment during which a screening pure-tone audiogram (only air conduction) is administered by the hearing aid dispenser. If the hearing loss in one or both ears is at least 35 decibel $(\mathrm{dB})$ hearing level (HL) (averaged over the three frequencies 1,2 and $4 \mathrm{kHz}$ ) in one or both ears, someone is considered potentially eligible for hearing aid fitting and more comprehensive audiometry is required. If the client is interested in hearing aids, his/her general wishes and goals are discussed after which the Amsterdam Inventory for Auditory Disability and Handicap (AIADH; Kramer et $a l)^{45}$ is handed out. Clients are asked to complete the AIADH at home and bring it along to the next appointment. The AIADH assesses hearing activity limitations and participation restrictions. Clients are asked to assign a CP and involve them throughout the rehabilitation (eg, bring them to appointments). During the next appointment, that is, the intake appointment, comprehensive audiometry (air and bone conduction, and speech audiometry) are performed. The results of all tests, the AIADH and the wishes of the client determine what type of hearing aid may be best suited for this person. The appropriate hearing aids will be selected and fitted directly (if available in the HAD practice) or in a subsequent fitting appointment. Fitting is followed by a trial 
period which usually lasts up to 4 weeks, during which people can try out the hearing aid and decide whether or not to purchase it. Depending on the client's needs, finetuning or other follow-up appointments are scheduled. These can be scheduled during the trial period and after the device has been purchased.

\section{Intervention: SUPR}

SUPR consists of a Practical Support Booklet and online elements. In addition, clients are asked to assign a CP who is involved actively in the programme (see below).

\section{Practical Support Booklet}

The Practical Support Booklet will be handed out at the end of the preparation appointment (first-time HAUs, experienced HAUs) or the intake appointment (experienced HAUs). The aims of the Practical Support Booklet are to: 1) assist clients and CPs in getting familiar with their hearing aid, 2) stimulate clients' use of the hearing aid and clients' and CPs' use of communication strategies and 3) guide clients and their CPs through the various stages (ie, appointments) of the rehabilitation trajectory. Although the theoretical elements of the booklet can also be used as a reference after the purchase of the hearing aid, the booklet's focus is on the period between the first HAD appointment and the end of the trial period. The booklet covers four parts, corresponding to the four key appointments during the trial period (ie, preparation appointment, intake appointment, control-tuning and/ or fine-tuning appointment and purchase appointment). The information that is provided is synchronised with the topics which are typically discussed during these appointments. The first part outlines the process of getting a hearing aid and includes an introduction to the hearing aid dispenser's care and an explanation about the pure tone audiogram. The client is asked to write down and rank specific communication goals (s) he wishes to reach by the end of the trial period (eg, "I want to be able to hear the stories of my 10 year old granddaughter Anne when I pick her up from school every Monday". The second part revolves around the types of hearing aids available and the client's hearing aid preferences. Information about how to operate and maintain the device is provided as well. In the third part, the client and the CP are asked to write down their experiences with the new hearing aid and its settings. This information will be used for further refinement of the fitting. The final section of the booklet provides information on assistive listening devices, reimbursement of costs, more information on the audiogram, types of hearing loss and the types of hearing aids (eg, behind-the-ear, in-the-canal or receiver-in-the-ear). In addition, an overview of the most important communication strategies that clients and their CP can apply is provided. The content and the appearance of the booklet were developed over the course of several months by the HAD company. Although no specific guidelines were used for the development of the written health information in the booklet, a number of the subsequent steps that are deemed important by Caposecco et al were taken into consideration: 1) interviews with key stakeholders (clients, CPs, HAD practice personnel) were held to specify the booklet's goals and functions, 2) graphics and text were developed and optimised with regard to their understandability and attractiveness (language difficulty, layout, font size, paragraphing), 3) a first complete version of the booklet was pilot-tested in $10 \mathrm{HAD}$ practices for several months. Feedback by all key stakeholders was collected and 4) the feedback was incorporated in a new and final version of the booklet (which was used in the study). ${ }^{46}$

\section{Online elements}

After the intake appointment, the links to the online elements will be sent to the participants via email. There are 2 emails which offer contact with the HAD practice and 11 emails which contain the links to the various educational videos that are offered (see below). The online part spans a period of up to about 6 months after the hearing aid purchase. The exact duration of SUPR depends on the duration of the trial period. For example, if a trial period is finalised in 3 weeks instead of the average four, the total duration of SUPR is 1 week shorter.

The educational videos consist of: 1) training modules on hearing aid handling skills. These comprise three short instruction videos with practical information on the use and maintenance of hearing aids. Participants receive the link to the relevant instruction video depending on their style of hearing aids (ie, behind-the-ear, in-the-canal or receiver-in-the-ear); 2) training modules on communication strategies and personal adjustment. This is a remake (ie, a modernised version) of the home educational programme 'Horen en Gehoord Worden: Hoe kan het beter', as developed by Kramer et al. ${ }^{37}$ It comprises five short videos showing the difficulties that hearing-impaired people can experience in everyday listening situations. The typical reactions by both the hearing-impaired people and his/ her social environment to these situations are shown, and a trainer illustrates how communication could be improved by using communication strategies (for both hearing-impaired people and his/her CP); 3) three testimonials by hearing-impaired peers who share their experiences with hearing aids.

\section{Measurements}

For all participants four measurements will beconducted: at baseline (after the preparation appointment, but before the actual hearing aid fitting) (T0), 6 months after the hearing aid purchase (T1), lyear after the hearing aid purchase (T2) and 18 months after the hearing aid purchase (T3). Measurements at T3 serve to determine the long-term effects of SUPR, that is, 1year after its completion. Data will be collected using online questionnaires through Survalyzer, which is an online survey programme. Email reminders will be sent within a week after the first invitation-email and another week after the first reminder, if necessary. 


\section{Study population and recruitment}

The following procedures were followed during the recruitment period (February 2016 to September 2016). Hearing aid dispensers invited clients to participate in the study. First-time HAUs were invited at the end of their preparation appointment. Experienced HAUs were invited at the end of their preparation or at the end of their intake appointment, if they did not require a preparation appointment. Hearing aid dispensers handed out an information package including an invitation letter, a selection form outlining the inclusion and exclusion criteria, a brochure about the study and an envelope with an information letter and brochure for the CP. All interested participants were asked to enrol themselves for the study by subscribing on a registration webpage and signing the online consent from there. Every month, the number of clients who were invited (number of envelopes that was handed out) and were enrolled (number of online subscriptions) per HAD practice were determined. When enrolment numbers for a particular HAD practice were relatively low, a phone call was made to the specific HAD practice to notify them of their current number of enrolments, to identify possible underlying reasons, and to motivate them to reach the required target. Throughout the recruitment period, the HAD headquarters organised motivational conference calls for the HAD practices that had not yet reached their target. Finally, when enrolment ratings continued to be behind target, employees of the headquarters directly invited potentially eligible clients who were not invited by the HAD practice personnel, via a telephone call. The information package was then sent via mail.

\section{Incentives}

After completing the T0 questionnaire, all participants will be offered a voucher of $€ 50$ to spend on a hearing aid or $€ 25$ to spend on other articles of the HAD practice if they decide not to purchase a hearing aid. CPs will be offered a gift card. In addition, participants in the control group will be offered a shortened version of SUPR after 18 months. For them, SUPR will be slightly adjusted such that it became suitable for individuals who had already started using a hearing aid.

Employees of the HAD practices will beoffered gift cards once the total number of participants is recruited (see ‘Sample size calculation' section).

\section{Inclusion criteria}

The following inclusion criteria for the hearing aid candidates were applied:

1) age 50 years or older; 2) willing to try out one or two new hearing aid(s) (ie, agreed to plan a follow-up appointment). This hearing aid could be their first (ie, first-time HAUs) or a replacement hearing aid (ie, experienced HAUs). Clients who did not purchase a hearing aid after the trial period were considered dropouts; 3) sufficient understanding of the Dutch language; 4) access to a personal computer with internet access and owner of an email account for the total duration of the study.

\section{Exclusion criteria}

The following hearing aid candidates were excluded: 1) candidates who received additional care at a specialised Audiology Clinic. In the Netherlands, an Audiology Clinic offers elaborate, multidisciplinary and specialised, tertiary healthcare and is aimed at people with complex hearing problems. This care may overlap and/or interfere with that of SUPR; 2) candidates who received a hearing aid primarily to suppress tinnitus complaints. For these individuals, the focus of the rehabilitation is not on restoring communication per se, and as such, they were not part of the target group of SUPR.

Although all participants were encouraged to assign a $\mathrm{CP}$, it was not obligatory for them to assign one in order to participate in the study. For the CPs, the only inclusion criterion applied was that they should be 18 years or older.

\section{Outcome measures}

An overview of all outcome measures and measurements over time according to Standard Protocol Items; Recommendations for Interventional Trials is attached (see online supplementary appendix 1$).{ }^{47}$

Primary outcome measures-HAUs

- The use of communication strategies will be measured using the reliable and validated Dutch 35-item version of the Communication Profile for the Hearing Impaired (CPHI) ${ }^{48} 49$ Communication strategies are measured using the following subscales: maladaptive behaviours, verbal strategies and non-verbal strategies. Each subscale consists of statements for which the respondent has to indicate how often (s) he applies this strategy. An example: "I avoid conversations with strangers, because of my hearing loss" (subscale maladaptive behaviour). The five response options range from 'almost never' to 'almost always'. Scores are averaged per subscale and range from 1 to 5 . Some items were recoded because of reverse scaling. High scores indicate favourable strategies, whereas low scores indicate unfavourable strategies.

We have chosen for the communication strategies subscales of the CPHI as central outcome measures for the following reasons. First, the subscales are purported to measure the constructs that are acted on by the core active element of the intervention (ie, the revised home education programme). Second, the CPHI has proven to have very good validity and reliability in the target population of this study. ${ }^{49}$

Secondary outcome measures-HAUs

- Personal adjustment to hearing impairment will also be measured using the reliable and validated Dutch 35item version of the CPHI. ${ }^{48} 49$ This second section of the CPHI deals with personal adjustment and also contains three subscales: self-acceptance, acceptance of loss and stress and withdrawal. An example item of 
the latter subscale is: "I feel very tense because of my hearing loss". The five response options range from 'totally disagree' (one) to 'totally agree' (five). All items were recoded because of reverse scaling. After recoding the item scores, average scores per subscale can be calculated, with low scores indicating poor personal adjustment and high scores indicating good personal adjustment.

- Self-efficacy of hearing aid handling will be measured by the basic handling subscale of the Measure of Audiologic Rehabilitation Self-Efficacy for Hearing Aids (MARS-HA). The English version of this 7-item subscale has good psychometric quality. ${ }^{50}$ Scores can range from $0 \%$ to $100 \%$, with lower scores representing less certainty in one's capability of handling a hearing aid. At T1, T2 and T3, the 5-item subscale advanced handling will be additionally administered. Dutch versions of the scales were created using the forwardbackward method. ${ }^{51}$ At T0 'expected self-efficacy' will be administered, whereas at $\mathrm{T} 1, \mathrm{~T} 2$ and $\mathrm{T} 3$ 'experienced self-efficacy' will be determined as the new hearing aids will have been fitted by then. For measurement of 'expected self-efficacy', all MARSHA-items start with "I think I can ...", whereas for measurement of "experienced self-efficacy all items start with "I can...".

- Hearing aid use. Self-reported use will be measured using the first item of the International Outcome Inventory-Hearing Aids (IOI-HA) ("How many hours per day on average have you been using your hearing aid(s) in the last twoweeks?"). Response options are 'none', 'less than 1 hour a day', ' $1-4$ hours a day', '4-8hours a day' and 'more than 8 hours a day' ${ }^{52}$ Self-reported hearing aid use will additionally be measured by three questions from the use questionnaire developed by Laplante-Lévesque $e t ~ a l^{53}$ The latter questionnaire was translated into Dutch, using the forward-backward method. ${ }^{51}$ Hearing aid use will also be measured objectively via data-logging.

- Self-reported intervention outcomes (hearingaidrehabilitation and SUPR outcome). IOI-HA (items 2-7) and the equivalent IOI-AI (all seven items) will be used to assess the outcome of hearing aid rehabilitation and SUPR, respectively. ${ }^{52}$ The Dutch version of IOI-HA has a good test-retest reliability and validity. ${ }^{54}$ The first item of the IOI-AI determines the frequency of the use of the alternative intervention, that is, "How often have you used the learnt communication strategies on an average day in the last 2 weeks?" Response options are 'never' (1), 'rarely' (2), 'sometimes' (3), 'often' (4) and 'almost always' (5). Items 2-7 of the IOI-HA/ IOI-AI questionnaire cover: benefit, residual activity limitations, satisfaction with the hearing aid(s)/ SUPR, residual participation restrictions, impact on others and quality of life.

- Satisfaction with the HAD practice service. Satisfaction will be measured by the following question: "How likely is it that you would recommend the service of the HAD practice to other people (family, friends, colleagues)?" It is scored on a visual analogue scale running from 0 (=not at all likely) to 10 (=extremely likely).

- Self-reported activity limitations and participation restrictions are measured using the reliable and validated original (Dutch) version of the AIADH. ${ }^{45}{ }^{55}$ It contains 28 questions regarding everyday listening situations. An example is: "Do you immediately look into the right direction when somebody calls you in the street?" The 4-point response scale covers: 'almost never' (1), 'sometimes' (2), 'often' (3) and 'almost always' (4). When the participant answers the question with 'almost never' or 'sometimes', he or she is directed to a next question, which is about the inconvenience of not being able to hear well in that specific situation. Response options are: 'no' (1), 'a little' (2), 'very handicapped' (3) and 'extremely handicapped' (4). Hence, the total score can range from 28 to 112 with higher scores indicating greater participation restrictions.

- Readiness to do something about one's hearing problems will be measured by the validated Dutch 24-item version of the University of Rhode Island Change Assessment. ${ }^{56}$ Formulations of items were adjusted such that they applied to hearing problems. The inventory contains 24 statements regarding attitudes and behaviours assessing an individual's stage of behaviour change. At $\mathrm{T} 0$, the scores on the following stages will be assessed: pre-contemplation (does not intend to take action in the foreseeable future, eg, "As far as I'm concerned, I don't have any problems with my hearing that need changing"), contemplation (intends to change in the next 6 months and is aware of the pros and cons of changing), and action (has made specific modifications in his/her lifestyle towards healthy behaviour). At T1, T2 and T3, the maintenance stage (can maintain the changes in new behaviour) will be added. The five response options range from 'fully disagree' (score one) to 'fully agree' (score five). Summed scores for each subscale will be calculated. In addition, the composite 'readiness score' (adding the contemplation, action and maintenance scores and subtracting the precontemplation score) and the composite 'committed action score' (subtracting the contemplation stage score from the action stage score) will be calculated. ${ }^{56}$ The higher the composite scores, the further the respondents are along the stages of change.

- Emotional response to hearing problems. The Hearing Handicap and Disability Inventory will be used. ${ }^{57}$ The purpose of the inventory is to identify the individual's problems caused by hearing loss. Only the section 'emotional response' will be administered. It contains five statements each with five response options: 'yes!' (4), 'yes' (3), 'more or less' (2), 'no' (1) and 'no!' (0). An example is: "I find it difficult to accept that I am hearing impaired". Lower scores indicate better outcomes. 
Secondary outcome measures-CP

- Third-party disability will be measured using the Significant Other Scale for Hearing Disability. ${ }^{12}$ This questionnaire was translated into Dutch for the purposes of this study following a forward-backward method. ${ }^{51}$ The 27 -item questionnaire addresses the problems and limitations experienced by the CP. An example item is: "Because of my partner's hearing difficulties I have to repeat myself often". For each item, the CP has to indicate how much of a problem it is for him/her: 'no problem' (0), 'a mild problem' (1), 'a moderate problem' (2), 'a severe problem' (3), 'a complete problem' (4). Higher scores indicate greater difficulties.

- Hearing aid rehabilitation and SUPR outcome as viewed from the perspective of the $C P$ will be administered with the 7-item IOI-HA-SO/IOI-AI-SO and covers use, benefit, residual activity limitations, satisfaction, residual participation restrictions, impact on others and quality of life. ${ }^{58}$

Baseline measurement-demographical characteristics

- Gender (male/female).

- Age (in years).

- Marital status (married/cohabiting/widow or widower/divorced/single, never married).

- Living situation (living together with my partner/ living together with my partner and children/living together without my partner but with one or more family members/living alone (own room) or in a care institution/living alone, independently or nursing home/other, namely...).

- Level of education (no completed education/lower general education, elementary education or a part of it/lower general secondary education/vocational education/secondary education/technical and vocational education/higher professional education/ higher general education/scientific education/other, namely...).

- Occupational status (yes/no).

- Country of birth (The Netherlands/other, namely...).

- Country of birth father (The Netherlands/other, namely...).

- Country of birth mother (The Netherlands/other, namely...).

- Hearing loss in each ear, in dB HL (averaged over 1, 2 and $4 \mathrm{kHz}$ ) as retrieved from the pure-tone audiogram as provided by the hearing aid dispenser.

\section{Randomisation}

$\mathrm{HAD}$ practices were randomly assigned to offer $\mathrm{CaU}$ or the intervention. To avoid an unequal distribution of HAD practices with regard to level of urbanisation, HAD practices were prestratified (HAD practices located in a relatively rural area vs in an urban area) and randomisation occurred within these two strata. A statistician performed block randomisation of the HAD practices in the statistical software $\mathrm{R}$, with random permutation in blocks of size four and with a fixed seed. Thirty-four HAD practices were assigned to $\mathrm{CaU}$ and $36 \mathrm{HAD}$ practices to the intervention group. The recruitment procedure and period was the same for all 70 included HAD practices (the total list of included HAD practices are available on request from the research team).

\section{Sample size calculation}

Sample size calculations are based on the expected effects of the intervention on the primary outcomes: communication strategies (CPHI). Demorest and Erdman indicated that the expected difference on the subscales of the CPHI varies from 0.67 (maladaptive behaviour) to 0.95 (self-acceptance) ${ }^{59}$ Given that in a previous study, ${ }^{37}$ the effect of the programme was larger for first-time than for experienced users, we calculated sample sizes separately for first-time and experienced users. For first-time HAUs, we based our sample size calculations on an expected difference of 0.67 between the intervention and the $\mathrm{CaU}$ group. Note that the subscale with the smallest minimal importance difference (ie, maladaptive behaviour) was used in the calculation, as finding a significant difference on this measure requires the largest number of participants. Calculations in PASS 12 (tests for two means in a cluster-randomised design; intracluster correlation coefficient: 0.01; alpha: 0.05; power: 0.80 ) shows that when 70 HAD practices are included (of which half will offer SUPR and half will offer $\mathrm{CaU}$ ), the number of first-time HAUs to include in the analyses is two per HAD practice. For the sample size calculation of the experienced users, we chose an expected difference of 0.4 between the intervention and CaU group. The expected difference was set lower than for first-time HAUs as Kramer $e t$ al had previously found generally smaller effects for experienced users than for first-time users. ${ }^{37}$ With a difference of 0.4 , the number of experienced HAUs (power: 0.80 ) to include is three per HAD practice. We expected the proportion of dropout or loss to follow-up across the study to be $20 \%$. This includes loss to follow-up for a range of reasons: no motivation anymore, reluctance to purchase a hearing aid after a successful trial, sickness, death, etc. Taking the loss to follow-up and the proportion of clients that normally purchase a hearing aid into account results in a total (rounded) number of four first-time HAUs per HAD practice and five experienced HAUs per HAD practice to be recruited.

\section{Statistical analyses}

To check the comparability between the groups (CaU or intervention group) at baseline, baseline characteristics of the participants will be compared using the $\chi^{2}$ test (for categorical variables), the independent samples t-test (for normally distributed continuous variables) and the Mann-Whitney U test (for non-normally distributed continuous variables). Comparability will be checked for all demographic variables and all primary and secondary outcomes. 
For the effect analyses, the groups will be compared on all primary and secondary outcome measures using linear mixed models including the results at T0, T1, T2 and T3. Group, time and their two-way interaction will be included as fixed effects in the mixed models, with random intercepts for subject and HAD practice. For the covariance matrix, a variance component structure will be chosen. To adjust for potential bias associated with multiplicity of analyses, statistical significance levels will beset at $\mathrm{p}<0.016(0.05 / 3)$. If a significant effect will be found, an independent samples t-test was used and a Bonferroni correction will be administered because of multiple comparisons. Type of HAU (first-time or experienced) will be tested as an effect modifier for potential subgroup differences.

In case of substantial missing data, multiple imputation will be applied. The main analysis was intention to treat. Any outcome measure to be collected for participants who discontinue or deviate from intervention protocols will be saved and analysed according to the intentionto-treat protocol. In addition, a per-protocol analysis will be performed. A per-protocol analysis includes those participants who completed the intervention originally allocated as described in the study protocol. As a per-protocol analysis can potentially yield biassed effects (eg, see CONSORT statement) ${ }^{60}$ great caution will be exerted when interpreting these results. In addition, the report of these findings in future articles will be nuanced explicitly and thoroughly.

\section{Process evaluation}

The process of implementing SUPR into the HAD care in the intervention arm will be evaluated. The main aims of this evaluation are to gain insight into 1) the circumstances in which the intervention was implemented, 2) (non-) compliance with the intervention and 3) the professionals' and clients' appraisal of the intervention.

The process evaluation will be carried out according to the framework as proposed by Linnan and Steckler. ${ }^{61}$ It covers seven parameters: recruitment, reach, fidelity, dose delivered, dose received and implemented, satisfaction and perceived benefit. ${ }^{62} \mathrm{~A}$ brief description of each of the parameters is given below.

- Recruitment refers to the procedures applied to approach and attract potential participants. The hearing aid dispensers will be asked to provide this information.

- Reach. This is the proportion of people participating relative to the number of people invited.

- Fidelity relates to the question of whether the intervention was provided as intended. The team that is responsible for the email contact will be asked to provide a written report on this.

- Dose delivered: 1) Did the personnel of the HAD practice hand out the Practical Support Booklet at the end of the preparation appointment? 2) Did the personnel of the HAD headquarters send out the emails correctly (correct content) and on time?
- Dose received and implemented: 1) Did the participants receive and use the Practical Support Booklet? 2) Did the participants open the emails and the videos? If so, did they watch the whole video, or part(s) of it? The video watching behaviour will be determined using Quadia (supplier of online video content) and Google analytics. Data on the average watching time per video, and how many times a particular video has been opened will be determined. Due to the privacy regulations the HAD company is subject to, the company is only allowed to collect video watching data on a group level (and not on an individual level). As all the HAD practices of the company that do not participate in the study provide SUPR as their standard care at the time of the study, the researchers will not be able to determine specific group averages of the study participants (the averages are based on both study participants and regular HAD clients). Information on implementation of the knowledge that participants learnt from SUPR will be deduced from the IOI-AI questionnaire (item on use) on T1. If participants received and used the Practical Support Booklet will be measured by a questionnaire.

- Satisfaction: satisfaction of the participant with SUPR will be evaluated using the IOI-AI questionnaire (item satisfaction) on $\mathrm{T} 1$. The hearing aid dispensers will be asked to answer the question: How would you rate your satisfaction with SUPR?

- Benefit: information on the experienced benefit of the participant will be obtained from the IOI-AI questionnaire (item benefit) on T1. The hearing aid dispensers will be asked to answer the question: How would you rate the perceived benefit from SUPR for your clients' ability to improve in communication?

Additionally, focus group discussions with participants from the intervention group will be organised to gain insight into the reasons for using the knowledge of SUPR in their daily lives or not. A minimum of two focus groups will be organised. The exact number will depend on data saturation. Heterogeneity in age, gender, educational level, severity of hearing impairment and stage of behaviour change (at baseline) within the groups will bestrived for. Given the difficulties hearing-impaired individuals might have with group conversations, the focus groups will have a maximum size of six participants each.

\section{ETHICS AND DISSEMINATION}

Protocol amendments, confidentiality and dissemination policy

Any future protocol modifications will be submitted to the VU University Medical Center Medical Ethical Committee. Directly on approval, the modification will be corresponded to the trial registry.

Personal information about enrolled participants will only be shared with employees of the headquarters of the HAD practices who signed a privacy declaration. This 
exchange of personal information will only occur in order to collect data within the framework of the study (eg, to collect audiogram data, hearing aid purchase status and use of SUPR). Any exchanged data and personal information will be password protected.

VU University Medical Center has all property rights on the final results of the trial and is entitled to publish the results. The funder is not entitled to publish the results without written consent of the VU University Medical Center. These agreements are secured in a contract. For specific author contributions for the current paper, see 'Authors' contributions'.

Findings of the study will be published in scientific journals and presented at scientific conferences, and were communicated within the national and international media. A short report of the study findings was sent to interested participants. The results were communicated within the hearing aid dispenser company.

\section{Data collection forms and data storage}

Data collection forms and procedures for data management are available on request. All data will be collected digitally and will be stored on a computer disk at the VU University Medical Center, which is locked with a security code only available to members of the SUPR research team. According to Good Clinical Practice guidelines and after having received informed consent, data will be archived for a period of 15 years after finalising the study. After finalisation, the key file (connecting participant numbers to the names and contact details of the participant) will be destroyed once it was expected that participants do not need to be approached further for the purposes of the study. We will perform double data entry of a selection of the audiograms and the baseline AIADH data for quality purposes.

\section{Monitoring}

The study is subjected to local regulations and its quality is monitored by the research institutes (ie, $\mathrm{EMGO}^{+}$) Quality Committee. This committee is responsible for developing, implementing and maintaining a system for quality assurance and control for all research within the institute. Due to the decision of the Dutch Institutional Review Board of the VU Medical University Center Amsterdam that the study does not fall under the Research Involving Human Subjects Act, the formation of a Clinical Trial Data Monitoring Committee was not deemed necessary.

\section{DISCUSSION}

Like in most parts in the world, usual care for adults with hearing impairment in the Netherlands is mostly restricted to audiological assessment and hearing aid fitting. This type of care is in the large part provided by commercial hearing aid dispensers. Communication programmes aimed at improving the use of favourable communication strategies, increasing personal adjustment to hearing impairment and improving hearing aid handling skills are not provided on a large scale in standard hearing healthcare settings. This is undesirable, as there is a growing body of evidence showing that offering such programmes can effectively decrease communication problems and associated negative health outcomes. ${ }^{27} 333842$ Likewise, despite the fact that including CPs in the rehabilitation process is increasingly recognised within audiology as a prerequisite for successful rehabilitation, ${ }^{12} \mathrm{CPs}$ are not yet part of standard hearing healthcare. In the current study, these elements (ie, a communication programme and involvement of a CP) are part of a programme called SUPR that is incorporated in regular hearing aid dispensing care and that will be tested for its effectiveness. SUPR's primary aims are to improve older hearing aid owners' communication strategies and personal adjustment and decrease their CPs' third-party disability. To our knowledge, similar online support programmes for HAUs that are implemented on a large scale in hearing aid dispenser settings are not yet available.

A strength of the SUPR programme is that for those who are at risk for isolation or those who have reduced access to healthcare, the internet can be a practical tool providing direct access to health services. ${ }^{63}$ Other elements that can add to the effectiveness of online support programmes as SUPR are that it can (partly or mainly) be provided in a visual mode (images, written text, subtitles), the volume can be controlled, background noises can be relatively easily eliminated and online support programmes provide the opportunity to tailor intervention elements.

A few limitations to the design need to be considered. Unfortunately, it is not possible to perform a doubleblinded, randomised controlled trial due to the nature of the intervention study. Blinding of the participants is not possible as they will be informed about the general aim of the SUPR study (ie, to evaluate a support programme) and know that they are either part of the group that receives CaU or SUPR. Nevertheless, we will attempt to minimise the provision of information on the content of SUPR to participants of the CaU group. The participants only know that SUPR is a support programme aimed to 'improve communication', but do not know what the intervention further entails. This way, we aimed to prevent that they would independently seek access to SUPR (which would cause contamination) and that their knowledge of the care they were missing out on would affect their responses in the questionnaires. We further attempted to prevent contamination by offering the programme to the $\mathrm{CaU}$ participants for free after completing the study. Blinding the researchers during the effect analysis is also not possible as the IOI measure that is administered at T1, T2 and T3 indicates what group each participant was randomised to (IOI-HA only: CaU group; IOI-AI: intervention group).

SUPR is an online intervention, it is thus essential that people have access to a device with internet access and an email account. Participants who have access to the internet will most likely be of high SES and this might bias the data. The fact that the support programme as 
such reaches a selective part of the dispenser's clientele requires further discussion. Consistent with findings from Choi and Dinitto and Fox, who compared non-internet users and users, it is possible that the older people participating in the SUPR study generally have a somewhat higher socioeconomic status and are somewhat younger than the average clientele of the dispenser. ${ }^{64}{ }^{65}$ With regard to age however, it should be noted among the young-old (aged 55-74 years) the weekly internet use has increased from $70 \%$ in 2010 to $83 \%$ in 2015 in the Netherlands and will most probably keep rising in the future. ${ }^{66}$ This suggests that the large majority of the younger-old can currently already be reached with SUPR and this will improve even more in the future. The non-use of internet among the older olds (aged 75+ years) currently still is substantial, although this proportion also has decreased strongly in the past few years $(66 \%$ in 2012 to $50 \%$ in 2015) ${ }^{67}$ Furthermore, it is encouraging that older internet users, generally use it more for health-related tasks or information than for personal tasks. ${ }^{68}$ In addition, people with hearing loss are more likely to use the internet than people in the general population $(\mathrm{OR}=1.74,95 \% \mathrm{CI} 1.23$ to 3.17) ${ }^{69}$ Baring these developments in mind, we are confident that the large majority of the older HAUs who can potentially benefit from SUPR will be increasingly eligible and open to using SUPR to improve their hearing health.

At the start of the study, participants might downplay their hearing problems because hearing loss stigma causes them to be reluctant to acknowledge or recognise their hearing problems. ${ }^{70}$ We expect that SUPR will have a positive effect on acceptation of hearing loss, and therefore people may report a disability level that is 'more honest'. This may hold particularly for the firsttime HAUs who have never gone through an intensive rehabilitation trajectory before and less so for the experienced users. As such, it is possible that this mechanism will cause an increase in self-reported hearing disability in the intervention group over time. This would counteract the favourable effect that SUPR is expected to create, that is, a decrease in experienced disability. To examine whether the first-mentioned mechanism would apply, one of the subscales of the CPHI on acceptation of hearing loss can be used.$^{48}$ With this subscale, we can examine if acceptance is a mediator between time and hearing status for the intervention group.

This study aims to perform a process evaluation, as is strongly recommended in all randomised controlled trial research. A process evaluation provides insight into reasons for the demonstrated (absence of) effectiveness of the intervention and might offer information concerning the generalisability of the study results. When no or only small significant effects of SUPR will be found, we may be able to modify the programme based on the results of the process evaluation after the study.

In the future, it is expected that there will be an increasing demand for solutions for hearing health conditions due to the ageing population and thus increased prevalence of hearing problems. SUPR is especially developed for use on a large-scale basis in HAD practices. The large number of practices that are involved in the study contributes to a large sample size (and statistical power), and it reflects real-world clinical practice. This will potentially make a strong case for the extrapolation of the study's results. Demonstrating the effectiveness of programmes would be a great step forward improving healthcare services for people with hearing impairment.

Correction notice This paper has been amended since it was published Online First. Owing to a scripting error, some of the publisher names in the references were replaced with 'BMJ Publishing Group'. This only affected the full text version, not the PDF. We have since corrected theseerrors and the correct publishers have been inserted into the references.

Acknowledgements We would like to acknowledge Schoonenberg B.V. (AudioNova International B.V.) for their contribution to the development of the SUPR study design and the writing of this paper.

Contributors SEK developed the first version of the study design in collaboration with BP and VJ. MP and SK developed the study design further and wrote up the first draft of the study protocol. BvdW, JFJM, MP, SEK and VJ worked on the design further and facilitated the practical implementation of the study. BIW provided statistical and methodological advice. Data collection will be done by BvdW and JFJM, assisted by VJ and supervised by SEK and MP. JFJM wrote the final version of the manuscript. SEK and MP supervised the writing process and MP, SEK, BP, BIW, VJ and BvdW gave critical comments on several drafts of the manuscript.

Funding The SUpport PRogramme study is funded by AudioNova International and is sponsored by the VU University Medical Centre. AudioNova International will have no role in the data analysis and/or the interpretation of the data.

Competing interests VJ is an employee at Schoonenberg Hoorcomfort. BP is an employee at AudioNova International.

Ethics approval Written consent for the SUpport PRogramme study (reference number: 2015.335) was obtained from the Dutch Institutional Review Board (IRB) of the VU Medical University Center Amsterdam (registered with the US Office for Human Research Protections as IRB00002991; FWA number: FWA00017598). The IRB concluded that Medical Research Involving Human Subjects ACT (WMO) does not apply to this study. Participants' consent will be obtained via the registration website of the study. At this website, participants were asked to declare that they were sufficiently informed about the study and agreed on the use of certain data to be collected for the purposes of the study.

Provenance and peer review Not commissioned; externally peer reviewed.

Data sharing statement It is not expected that participant level data will be made available because this has not been applied for in the ethics application. Approval has not been sought for the data to be publicly available.

Open Access This is an Open Access article distributed in accordance with the Creative Commons Attribution Non Commercial (CC BY-NC 4.0) license, which permits others to distribute, remix, adapt, build upon this work non-commercially, and license their derivative works on different terms, provided the original work is properly cited and the use is non-commercial. See: http://creativecommons.org/ licenses/by-nc/4.0/

(c) Article author(s) (or their employer(s) unless otherwise stated in the text of the article) 2017. All rights reserved. No commercial use is permitted unless otherwise expressly granted.

\section{REFERENCES}

1. Vos T, Barber RM, Bell B, et al. Global, regional, and national incidence, prevalence, and years lived with disability for 301 acute and chronic diseases and injuries in 188 countries, 1990-2013: a systematic analysis for the Global Burden of Disease Study 2013. Lancet 2015;386:743-800.

2. United Nations, Department of Economic and Social Affairs, Population Division. World population Ageing. New York: United Nations, 2015. 
3. Weinstein BE, Sirow LW, Moser S. Relating hearing aid use to social and emotional loneliness in older adults. Am J Audiol 2016;25:54-61.

4. Pronk M, Deeg DJ, Smits C, et al. Prospective effects of hearing status on loneliness and depression in older persons: identification of subgroups. Int J Audiol 2011;50:887-96.

5. Strawbridge WJ, Wallhagen MI, Shema SJ, et al. Negative consequences of hearing impairment in old age: a longitudinal analysis. Gerontologist 2000;40:320-6.

6. Nachtegaal J, Smit JH, Smits C, et al. The association between hearing status and psychosocial health before the age of 70 years: results from an internet-based national survey on hearing. Ear Hear 2009;30:302-12.

7. Saito $\mathrm{H}$, Nishiwaki $\mathrm{Y}$, Michikawa $\mathrm{T}$, et al. Hearing handicap predicts the development of depressive symptoms after 3 years in older community-dwelling Japanese. J Am Geriatr Soc 2010;58:93-7.

8. Nachtegaal J, Festen JM, Kramer SE. Hearing ability in working life and its relationship with sick leave and self-reported work productivity. Ear Hear 2012;33:94-103.

9. Lin FR, Yaffe K, Xia J, et al. Hearing loss and cognitive decline in older adults. JAMA Intern Med 2013:173:293-9.

10. Jiam NT, Li C, Agrawal Y. Hearing loss and falls: a systematic review and meta-analysis. Laryngoscope 2016;126:2587-96.

11. World Health Organization. International classification of functioning, disability and Health. Geneva: World Health Organization, 2001.

12. Scarinci N, Worrall L, Hickson L. The effect of hearing impairment in older people on the spouse: development and psychometric testing of the significant other scale for hearing disability (SOS-HEAR). Int $J$ Audiol 2009;48:671-83.

13. Kamil RJ, Lin FR. The effects of hearing impairment in older adults on communication partners: a systematic review. J Am Acad Audiol 2015;26:155-82.

14. Jennings MB, Shaw L. Impact of hearing loss in the workplace: raising questions about partnerships with professionals. Work 2008;30:289-95.

15. Chisolm TH, Johnson CE, Danhauer JL, et al. A systematic review of health-related quality of life and hearing aids: final report of the American Academy of Audiology Task Force on the Health-Related Quality of Life Benefits of Amplification in Adults. J Am Acad Audiol 2007;18:151-83.

16. Mulrow CD, Tuley MR, Aguilar C. Sustained benefits of hearing aids. J Speech Hear Res 1992;35:1402-5.

17. Acar B, Yurekli MF, Babademez MA, et al. Effects of hearing aids on cognitive functions and depressive signs in elderly people. Arch Gerontol Geriatr 2011;52:250-2.

18. Amieva H, Ouvrard C, Giulioli C, et al. Self-reported hearing loss, hearing aids, and cognitive decline in elderly adults: a 25-Year study. J Am Geriatr Soc 2015;63:2099-104.

19. Chia EM, Wang JJ, Rochtchina E, et al. Hearing impairment and health-related quality of life: the Blue Mountains Hearing Study. Ear Hear 2007;28:187-95

20. Hartley D, Rochtchina E, Newall P, et al. Use of hearing AIDS and assistive listening devices in an older Australian population. $J \mathrm{Am}$ Acad Audiol 2010;21:642-53.

21. Smits C, Kramer SE, Houtgast T. Speech reception thresholds in noise and self-reported hearing disability in a general adult population. Ear Hear 2006;27:538-49.

22. Gates GA, Cooper JC, Kannel WB, et al. Hearing in the elderly: the Framingham cohort, 1983-1985. Part I. Basic audiometric test results. Ear Hear 1990;11:247-56.

23. Abrams HB, Kihm J. An introduction to MarkeTrak IX: a New Baseline for the Hearing Aid Market. Hearing Review 2015;22:16.

24. Knudsen LV, Oberg M, Nielsen C, et al. Factors influencing help seeking, hearing aid uptake, hearing aid use and satisfaction with hearing aids: a review of the literature. Trends Amplif 2010;14:127-54

25. Meyer C, Hickson L. What factors influence help-seeking for hearing impairment and hearing aid adoption in older adults? Int J Audiol 2012;51:66-74

26. McCormack $\mathrm{A}$, Fortnum $\mathrm{H}$. Why do people fitted with hearing aids not wear them? Int J Audiol 2013;52:360-8.

27. Boothroyd A. Adult aural rehabilitation: what is it and does it work? Trends Amplif 2007:11:63-71.

28. Hickson L, Scarinci N. Older adults with acquired hearing impairment: applying the ICF in rehabilitation. Semin Speech Lang 2007;28:283-90.

29. Granberg S, Swanepoel de W, Englund U, et al. The ICF core sets for hearing loss project: International expert survey on functioning and disability of adults with hearing loss using the international classification of functioning, disability, and health (ICF). Int J Audiol 2014;53:497-506.
30. Granberg S, Pronk M, Swanepoel de W, et al. The ICF core sets for hearing loss project: functioning and disability from the patient perspective. Int J Audiol 2014;53:777-86.

31. Gagné JP, Jennings MB. Audiologic rehabilitation intervention services for adults with acquired hearing impairment. In: Valente $\mathrm{M}$, Hosford-Dunn H, Roeser RJ, eds. Audiology: treatment. New York: thieme Medical Publishers, 2008:370-99.

32. Laplante-Lévesque A, Hickson L, Worrall L. Factors influencing rehabilitation decisions of adults with acquired hearing impairment. Int J Audiol 2010:49:497-507.

33. Kiessling J, Pichora-Fuller MK, Gatehouse S, et al. Candidature for and delivery of audiological services: special needs of older people. Int J Audiol 2003;42(suppl 2):92-101.

34. Barker F, Mackenzie E, Elliott L, et al. Interventions to improve hearing aid use in adult auditory rehabilitation. Cochrane Database Syst Rev 2016;8:CD010342.

35. Henshaw H, Ferguson MA. Efficacy of individual computer-based auditory training for people with hearing loss: a systematic review of the evidence. PLoS One 2013;8:e62836.

36. Wong L, Hickson L. Evidence-based practice in audiology: evaluating interventions for children and adults with hearing impairment. San Diego, CA: Plural Publishing, 2012.

37. Kramer SE, Allessie GH, Dondorp AW, et al. A home education program for older adults with hearing impairment and their significant others: a randomized trial evaluating short- and long-term effects. Int J Audiol 2005;44:255-64.

38. Hickson L, Worrall L, Scarinci N. A randomized controlled trial evaluating the active communication education program for older people with hearing impairment. Ear Hear 2007;28:212-30.

39. Hickson L. Defining a Paradigm shift. Semin Hear 2012;33:003-8.

40. Lusis I, Mason P. Paradigm shift: the new world of hearing health care delivery. ASHA Lead 2012;17:36-7.

41. Tognola G, Paglialonga A, Chiaramello E, et al. eHealth for hearingnew views and apps practicalities. EJBI 2015;11:37-49.

42. Thorén $\mathrm{ES}$, Öberg $\mathrm{M}$, Wänström $\mathrm{G}$, et al. A randomized controlled trial evaluating the effects of online rehabilitative intervention for adult hearing-aid users. Int J Audiol 2014;53:452-61.

43. Thorén E, Svensson M, Törnqvist A, et al. Rehabilitative online education versus internet discussion group for hearing aid users: a randomized controlled trial. J Am Acad Audiol 2011;22:274-85.

44. Ferguson $\mathrm{M}$, Brandreth $\mathrm{M}$, Brassington $\mathrm{W}$, et al. A randomized controlled Trial to evaluate the benefits of a multimedia educational program for first-time hearing aid users. Ear Hear 2016;37:123-36.

45. Kramer SE, Kapteyn TS, Festen JM, et al. Factors in subjective hearing disability. Audiology 1995;34:311-20.

46. Caposecco A, Hickson L, Meyer C. Assembly and insertion of a selffitting hearing aid: design of effective instruction materials. Trends Amplif 2011:15:184-95.

47. Chan AW, Tetzlaff JM, Altman DG, et al. SPIRIT 2013 statement: defining standard protocol items for clinical trials. Ann Intern Med 2013;158:200-7.

48. Demorest ME, Erdman SA. Development of the communication profile for the hearing impaired. J Speech Hear Disord 1987;52:129-43.

49. Mokkink LB, Knol DL, van Nispen RM, et al. Improving the quality and applicability of the Dutch scales of the communication Profile for the hearing impaired using item response theory. J Speech Lang Hear Res 2010;53:556-71.

50. West RL, Smith SL. Development of a hearing aid self-efficacy questionnaire. Int J Audiol 2007:46:759-71.

51. Beaton DE, Bombardier C, Guillemin F, et al. Guidelines for the process of cross-cultural adaptation of self-report measures. Spine 2000;25:3186-91.

52. Kozlowski L, Almeida G, Ribas A. Level of user satisfaction with hearing AIDS and environment: the international outcome inventory for hearing AIDS. Int Arch Otorhinolaryngol 2014;18:229-34.

53. Laplante-Lévesque A, Nielsen C, Jensen LD, et al. Patterns of hearing aid usage predict hearing aid use amount (data logged and self-reported) and overreport. J Am Acad Audiol 2014;25:187-98.

54. Kramer SE, Goverts ST, Dreschler WA, et al. International Outcome Inventory for Hearing Aids (IOI-HA): results from the Netherlands. Int $J$ Audiol 2002;41:36-41.

55. Meijer AG, Wit HP, TenVergert EM, et al. Reliability and validity of the (modified) Amsterdam Inventory for Auditory Disability and Handicap. Int J Audiol 2003;42:220-6.

56. Laplante-Lévesque A, Hickson L, Worrall L. Stages of change in adults with acquired hearing impairment seeking help for the first time: application of the transtheoretical model in audiologic rehabilitation. Ear Hear 2013;34:447-57.

57. van den Brink RHS. Attitude and illness behavior in hearing impaired elderly (Unpublished doctoral thesis).Rijks University of Groningen, 1995. 
58. Noble W. Extending the IOI to significant others and to non-hearingaid-based interventions. Int J Audiol 2002;41:27-9.

59. Demorest ME, Erdman SA. Retest stability of the communication profile for the hearing impaired. Ear Hear 1988;9:237-42.

60. Moher D, Hopewell S, Schulz KF, et al. CONSORT 2010 explanation and elaboration: updated guidelines for reporting parallel group randomised trials. Int J Surg 2012:10:28-55.

61. Linnan L, Steckler A. Process evaluation for public health interventions and research. 1st ed. San Francisco CA: Jossey-Bass, 2002.

62. Gussenhoven AH, Singh AS, Goverts ST, et al. A process evaluation of implementing a vocational enablement protocol for employees with hearing difficulties in clinical practice. Int $J$ Audiol 2015;54:507-17.

63. Swanepoel deW, Hall JW. A systematic review of telehealth applications in audiology. Telemed J E Health 2010;16:181-200.
64. Choi NG, Dinitto DM. Internet use among older adults: association with health needs, psychological capital, and social capital. J Med Internet Res 2013;15:e97.

65. Fox S. Digital Divisions. PEW Internet \& American Life Project. Washington, DC, 2005.

66. UNECE Statistical Database. 2015 http://w3.unece.org/PXWeb/en (accessed 20 Jun 2016)

67. Centraal Bureau voor statistiek (CBS). 2016 https://www.cbs.nl/nl-nl/ nieuws/2016/22/acht-procent-van-de-nederlanders-nooit-op-internet (accessed 26 Sep 2016).

68. Gell NM, Rosenberg DE, Demiris G, et al. Patterns of technology use among older adults with and without disabilities. Gerontologist 2015;55:412-21.

69. Thorén ES, Öberg M, Wänström G, et al. Internet access and use in adults with hearing loss. J Med Internet Res 2013;15:e91.

70. Martin KA, Leary MR, Rejeski WJ. Self-Presentational concerns in older adults: implications for Health and Well-Being. Basic Appl Soc Psych 2000;22:169-79. 